\title{
The relationship between the apolipoprotein E e4 allele and hippocampal magnetic resonance imaging volume in community-dwelling individuals with mild Alzheimer's disease
}

This article was published in the following Dove Press journal:

Degenerative Neurological and Neuromuscular Disease

2 March 2013

Number of times this article has been viewed

\author{
Bernard Walsh' \\ Stuart Slater ${ }^{2}$ \\ Balakrishnan Nair ${ }^{3}$ \\ John Attia ${ }^{3}$ \\ 'Department of Geriatric Medicine, \\ John Hunter Hospital, Newcastle, \\ Australia; ${ }^{2}$ Hunter Imaging Group, \\ Cardiff, Australia; ${ }^{3}$ Centre for \\ Clinical Epidemiology and Biostatics, \\ University of Newcastle, Australia
}

Correspondence: Bernard Walsh Department of Geriatric Medicine, John Hunter Hospital, 2 Lookout Road, New Lambton Heights, Newcastle, NSW 2305, Australia

Tel +6I 2492 I4830

Email Bernard.Walsh@hnehealth.nsw.gov.au
Background: The degree of hippocampal magnetic resonance imaging (MRI) volume loss in Alzheimer's disease (AD) is commonly accepted as a marker of disease severity, yet remains expensive, unavailable, or not tolerated by many patients.

Aim: To examine whether the presence of one or more apolipoprotein E (ApoE) e4 alleles is associated with smaller hippocampal MRI volumes in a population of early AD patients.

Methods: A total of 88 consecutive patients attending a community-based memory disorders clinic who had both mild dementia on the Clinical Dementia Rating scale and Diagnostic and Statistical Manual of Mental Disorders criteria for probable AD were recruited. We examined the relationship between ApoE e4 allele load and hippocampal atrophy on MRI volumes.

Results: There was no association between the ApoE e4 load and hippocampal volume in this cohort.

Conclusion: This study suggests that the presence of one or more ApoE e4 alleles cannot be used to estimate pathological disease load in early AD.

Keywords: apolipoprotein E, Alzheimer's disease, MRI, hippocampus

\section{Introduction}

There is a lack of consensus regarding the utility of the apolipoprotein E (ApoE) genotype in the clinical management and prognostication of individuals with Alzheimer's disease (AD), where the ApoE e4 allele is reported to have multiple negative outcomes. As a cumulative and progressive neurodegenerative process, therapeutic intervention in the earliest stages of AD offers the most potential for preventing or slowing progression of the disease. The Clinical Dementia Rating (CDR) scale ${ }^{1}$ is both a useful and common tool for classification of dementia severity. It is used by many AD clinicians, but lacks the ability to predict the prognosis, rate of decline, response to drug therapy, or the onset of behavioral and psychotic symptoms. ApoE e4 has the potential to aid markedly in these clinical settings, but its optimal use has not been well defined. The degree of hippocampal magnetic resonance imaging (MRI) volume loss in AD is commonly accepted as a marker of disease severity, yet remains expensive, unavailable, or not tolerated by many patients. Examining the potential of the ApoE e4 allele as a simple, readily available substitute to MRI to infer the extent of hippocampal shrinkage has significant appeal, especially in the early stages of the disease.

ApoE is the major apolipoprotein species in the brain, and is involved in the catabolism of triglyceride-containing lipoproteins and the transport of these, fat-soluble 
vitamins and cholesterol. It is a $34 \mathrm{kDa}$ protein mediating the binding of lipoproteins to the low-density lipoprotein receptor (mainly on neuronal membranes), and it plays a key role in the mobilization of cholesterol and phospholipids during neuronal membrane remodeling associated with synaptic plasticity and neuronal repair. Also, there is evidence that ApoE isoforms alter the transport and metabolism of $A \beta$ amyloid in the brain. ${ }^{2}$

The possibility of a link between the ApoE genotype and the cross-sectional degree of medial temporal lobe atrophy on MRI in AD has been examined in relatively small studies in the last decade, with sample sizes varying between 15 and $49,{ }^{3-9}$ and the largest study having $138 \mathrm{AD}$ patients. ${ }^{10}$ The majority (six of eight) of these studies suggested a positive correlation between ApoE e4 and medial temporal lobe atrophy. One study suggested no difference across genotype in hippocampal volumes but significant atrophy in the adjoining amygdalae in the presence of an e4 allele. ${ }^{11}$ However in this study subjects appear to have been recruited from a mixture of settings, including hospitals and residential care facilities, and so did not predominantly concentrate on community-dwelling, independent subjects with early $\mathrm{AD}$, where such a genotype linkage would provide most clinical benefit for intervention. Also, inadequate definition of the varying AD severity makes interpretation of these findings difficult. ApoE e4 alleles in AD patients do however appear to be associated with increased rates of hippocampal volume loss over time. ${ }^{12}$

\section{Methods}

\section{Study population}

A total of 88 consecutive patients attending a communitybased memory disorders clinic who met the criteria for both Mild Dementia using the CDR scale and the Diagnostic and Statistical Manual of Mental Disorders (DSM-IV-TR) criteria for probable Alzheimer's disease ${ }^{13}$ agreed to take part in the study. These entry criteria were used in the clinic setting by a single, experienced geriatrician to identify eligible participants. The Mini-Mental Status Examination (MMSE) ${ }^{14}$ and age at diagnosis were consistent with this early stage of dementia (Table 1).

\section{Ethics approval}

The Hunter New England Human Research Ethics Committee approved this research and operates in accordance with the Australian National Health and Medical Research Council's National Statement on Ethical Conduct in Human Research (2007) and the Committee for Medicinal Products for Human Use/International Conference on Harmonisation
Table I Clinical characteristics of study population (88 CDR I.0 patients)

\begin{tabular}{llll}
\hline & $\begin{array}{l}\text { No e4 } \\
(3 \mathrm{I})\end{array}$ & $\begin{array}{l}\text { Single e4 } \\
\mathbf{( 4 6 )}\end{array}$ & $\begin{array}{l}\text { Double e4 } \\
\text { (II) }\end{array}$ \\
\hline Female (\%) & 14 & 26 & 10 \\
& $(45.2 \%)$ & $(56.5 \%)$ & $(91 \%)$ \\
Age at diagnosis & 77.4 & 77.3 & 71.8 \\
(mean, years) & & & \\
MMSE & 22.5 & 22.3 & 24.1 \\
(standard deviation) & $(3.3)$ & $(3.2)$ & $(2.9)$ \\
Nonsmokers (\%) & 29 & 43 & 11 \\
& $(93.5 \%)$ & $(93.5 \%)$ & $(100 \%)$ \\
\hline
\end{tabular}

Abbreviation: MMSE, mini-mental state examination.

of Technical Requirements for Registration of Pharmaceuticals for Human Use guidance on good clinical practice. No external funding was used in this study.

\section{ApoE genotyping}

All ApoE genotyping was performed in a single laboratory using polymerase chain reaction applied to whole blood lymphocytes.

\section{MRI hippocampal mapping}

All patients were imaged with 1.5 T (Signa Excite, General Electric Medical Systems, Milwaukee, WI, USA). All volume measurements were derived from $T_{1}$-weighted spoiled gradient-recalled echo sequence with $25^{\circ}$ flip angle, $1.6 \mathrm{~mm}$ slice thickness, $22 \times 19 \mathrm{~cm}$ field of view, TR 27, TE 9, and $320 \times 224$ matrix and 1.0 NEX.

All measurements were made by manual tracing of the coronal sections of the hippocampus and entorhinal cortices (ERC), and the volume measurements were calculated using the software provided by General Electric Medical Systems. The hippocampi were measured from the level of the mammillary body to the posterior margin of the quadrigeminal plate on the coronal images, which are considered constant markers on MRI. The ERC measurements were made on a single slice at the level of the mammillary body. A radiologist experienced in these measurements and who was blinded to the MMSE and ApoE status reported the MRI volumes.

\section{Statistics}

The statistical software package used was Stata version 11 (StataCorp, College Station, TX, USA). Assumptions of normality were checked for both age and sex distribution of the cohort. The independent variable was the ApoE e4 status (nil, 1 positive allele, 2 positive alleles) and the dependent variables were the MRI volume of the right and left hippocampi. Analysis of variance was used to test for differences in 


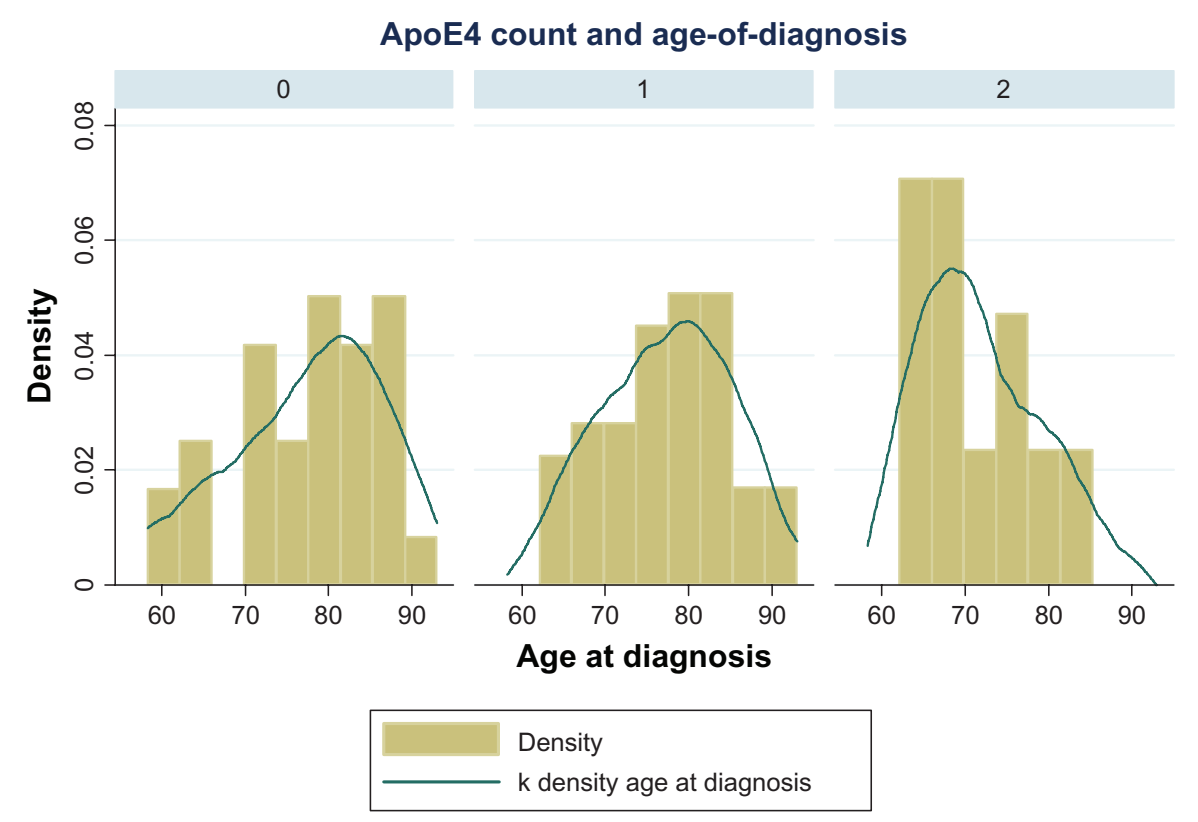

Figure I Age of Alzheimer's disease onset across apolipoprotein (ApoE) e4 status (0, I, and $2 \times$ e 4 alleles).

volume across the three allele groups. The measures of the ERC were excluded from the study analysis due to the lack of technically reliable measures of this structure.

\section{Results}

Table 1 provides the basic demographics of the three genotype groups. Participants were predominantly nonsmokers and had mean MMSE scores between 22 and 24. There was a predominance of younger and female participants in the e4/e4 group. This nonnormal distribution of age of onset across the three genotype groups is consistent with published literature that e4/e4 individuals have a 5- to 10-year earlier onset of Alzheimer's compared to the other genotype groups (Figure 1).

One-way analysis of variance was applied to test for any difference in hippocampal volume (left then right cortex) across the three allele groups. No differences between the mean volume measurements across the individual genotype groups were demonstrated (Figure 2) (left hippocampal volume, $P>0.86$; right hippocampal volume, $P>0.68$ ).

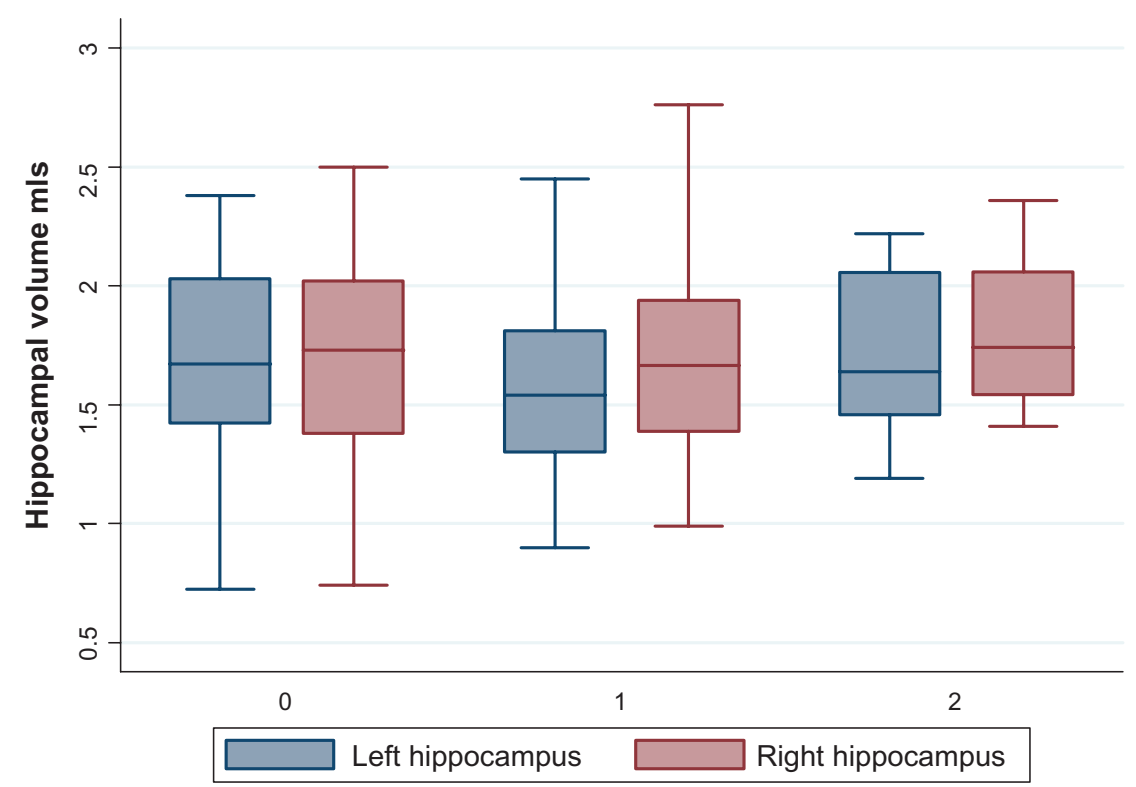

Figure 2 Left and right hippocampal volumes across apolipoprotein E e4 load ( 0 , I, and $2 \times$ e 4 alleles). 
Table 2 Multiple linear regression of hippocampal volume

\begin{tabular}{llllll}
\hline & \multicolumn{2}{l}{$\begin{array}{l}\text { Left hippocampal } \\
\text { volume }\end{array}$} & & \multicolumn{2}{l}{$\begin{array}{l}\text { Right hippocampal } \\
\text { volume }\end{array}$} \\
\cline { 2 - 3 } \cline { 6 - 6 } & Coefficient & P-value & & Coefficient & P-value \\
\hline Single e4 & -0.05 & 0.60 & & 0.02 & 0.80 \\
Double e4 & 0.002 & 0.99 & & 0.08 & 0.60 \\
Age at diagnosis & -0.02 & 0.001 & & -0.02 & 0.003 \\
Sex & -0.13 & 0.13 & & -0.16 & 0.06 \\
\hline
\end{tabular}

We also demonstrated that this lack of association between genotype and hippocampal volume remained when adjusted for age and sex using linear regression (Table 2).

\section{Discussion}

The study demonstrated no association between ApoE e4 status and hippocampal volume in early $\mathrm{AD}$ patients living in community settings, so confirming that the presence of one or more e 4 alleles does not estimate the degree of hippocampal volume loss in early $\mathrm{AD}$.

There were no significant differences in the distribution of baseline cognitive scores between the groups defined by ApoE status. As was consistent with previous literature, the expected difference in the age of onset of the double-e4 group compared to the single e 4 and non-e4 groups was demonstrated.

The strengths of this study are its unique community setting of early, well-defined AD patients where the clinical utility of ApoE genotype would be of most benefit. The large sample size and consecutive recruitment of these community dwellers increases the study's ability to reflect the true community AD ApoE e4 distribution. Also, all clinical assessments and disease-severity staging were performed by a single, senior geriatrician applying standard AD grading (CDR) and diagnostic criteria (DSM IV-TR), with a single observer conducting all MRI volume measurements blinded to both ApoE genotype and clinical details.

This study's results rely on the published literature's consensus of the correlation between AD pathological load and medial temporal lobe atrophy measured by MRI. If this correlation was disproved in the future, further studies into the possible association of ApoE e4 allele and early $\mathrm{AD}$ pathology would be required.

\section{Disclosure}

The authors report no conflicts of interest in this work.

\section{References}

1. Morris JC. The Clinical Dementia Rating (CDR): current version and scoring rules. Neurology. 1993;43:2412-2414.

2. Kim J, Basak J, Holtzman D. The role of apolipoprotein E in Alzheimer's disease. Neuron. 2009;63:287-303.

3. Baker R, Gholkar A, Scheltens P, et al. ApoE e4 allele, temporal lobe atrophy, and white matter lesions in late life dementias. Arch Neurol. 1999;56:961-965.

4. Geroldi C, Pihlajamäki M, Laakso M, et al. APOE-epsilon4 is associated with less frontal and more medial lobe atrophy in AD. Neurology. 1999;53:1825-1832.

5. Jack CR Jr, Petersen RC, Xu YC, et al. Hippocampal atrophy and apolipoprotein E genotype are independently associated with Alzheimer's disease. Ann Neurol. 1998;43:303-310.

6. Tanaka S, Kawamata J, Shimohama S, et al. Inferior temporal lobe atrophy and ApoE genotypes in Alzheimer's disease. X-ray computed tomography, magnetic resonance imaging and Xe-133 SPECT studies. Dement Geriatr Cogn Disord. 1998;9:90-98.

7. Juottonen K, Lehtovirta M, Helisalmi S, Riekkinen PJ Sr, Soininen H. Major decrease in the volume of the entorhinal cortex in patients with Alzheimer's disease carrying the apolioprotein E epsilon4 allele. J Neurol Neurosurg Psychiatry. 1998;65:322-327.

8. Mueller SG, Schuff N, Raptentsetsang S, Elman J, Weiner MW. Selective effect of Apo e4 on CA3 and dentate in normal aging and Alzheimer's disease using high resolution MRI at $4 \mathrm{~T}$. Neuroimage. 2008;42:42-48.

9. Lehtovirta M, Laakso M, Soininen H, et al. Volumes of hippocampus, amygdala and frontal lobe in Alzheimer's patients with different apolipoprotein E genotypes. Neuroscience. 1995;67:65-67.

10. Hashimoto M, Yasuda M, Tanimukai S, et al. Apolipoprotein E e4 and the pattern of regional brain atrophy in Alzheimer's disease. Neurology. 2001;57:1461-1466.

11. Basso M, Gelernter J, Yang J, et al. Apolipoprotein E epsilon4 is associated with atrophy of the amygdala in Alzheimer's Disease. Neurobiol Aging. 2006;27:1416-1424.

12. Schuff N, Woerner N, Boreta L, et al. MRI of hippocampal volume loss in early Alzheimer's Disease in relation to ApoE genotype and biomarkers. Brain. 2009;132:1067-1077.

13. American Psychiatric Association. Diagnostic and Statistical Manual of Mental Disorders, 4th ed, text revision. Arlington (VA): APA; 2000.

14. Folstein M, Folstein S, McHugh P. "Mini-mental state." A practical method for grading the cognitive state of patients for the clinician. J Psychiatr Res. 1975;12:189-198.
Degenerative Neurological and Neuromuscular Disease

\section{Publish your work in this journal}

Degenerative Neurological and Neuromuscular Disease is an international, peer-reviewed, open access journal focusing on research into degenerative neurological and neuromuscular disease, identification of therapeutic targets and the optimal use of preventative and integrated treatment interventions to achieve improved outcomes, enhanced

\section{Dovepress}

survival and quality of life for the patient. The manuscript management system is completely online and includes a very quick and fair peer-review system. Visit http://www.dovepress.com/testimonials.php to read real quotes from published authors. 